\title{
Lipopeptide producing endophytic bacteria of the genus Bacillus in the regulation of the expression of genes involved in the defense response of wheat against greenbug aphid Schizaphis graminum
}

\author{
Alekseev V.Y., Shein M.Yu., Veselova S.V., Burkhanova G.F., Maksimov I.V. \\ Institute of Biochemistry and Genetics of the UFRC of the RAS, Ufa, Russia \\ * email: valentin-1994@yandex.ru
}

Plant growth-promoting bacteria (PGPB), which produce various metabolites with antibiotic activity, are able to suppress the development of pests, both through direct antagonism and indirectly, through the mechanism of triggering induced systemic resistance (ISR). To date, the mechanism of action of bacterial lipopeptide on the plant defense system is just beginning to be studied. ISR induced by endophytic bacteria leads to a change in the redox status of plants and, as a result, to a change in the expression of genes encoding PR-proteins (from pathogenesis related proteins). Cellular factors such as Dicer-like (DCL) and Argonaute (AGO), which are involved in gene silencing, also induce systemic immunity to various pathogens through the regulation of $P R$ genes and their protein products. However, their role in these processes is not sufficiently studied. In our work, we studied three strains of endophytic bacteria Bacillus subtilis 26D, B. subtilis 11BM, and B. thuringiensis B-6066 from the All-Russian collection of industrial microorganisms, producing the lipopeptides surfactin, iturin, and fengicin respectively. And two B. subtilis Tas-1 and Tas-8.2 isolates excreted from the internal tissues of wheat plants. Both isolates produced surfactin. All bacterial strains and their metabolites exhibit insecticidal activity against the greenbug aphid $S$. graminum under direct exposure. During indirect action, the strains synthesizing surfactin and fengicin, but not iturin, had the greatest effect on the viability of greenbug aphid and the endurance of wheat plants. They also induced systemic resistance, which was manifested in the accumulation of hydrogen peroxide, increased activity of peroxidases, and the accumulation of transcripts of genes encoding PR-proteins markers of the salicylate and jasmonate signaling pathways $(P R-1, P R-2, P R-3, P R-6, P R-9)$ and genes of cellular factors DCL (DCL2, DCL4) and AGO (Agol, Ago2, Ago3).

Acknowledgments: This work was supported by the RFBR projects No. 20-316-90021 and 20-316-90004. 